\title{
Genetic interactions between the parasitoid wasp Leptopilina boulardi and its Drosophila hosts
}

\author{
A Dubuffet ${ }^{1}$, S Dupas 2 , F Frey ${ }^{2}$, J-M Drezen ${ }^{1}$, M Poirié $^{3}$ and Y Carton ${ }^{2}$ \\ ${ }^{1} I R B I$, Université François-Rabelais, Parc Grandmont, Tours, France; ${ }^{2}$ Laboratoire Populations, Génétique et Evolution, CNRS, \\ Gif-sur-Yvette Cedex, France and ${ }^{3} I N R A$, Université de Nice-Sophia Antipolis, Sophia Antipolis, France
}

\begin{abstract}
Coevolutionary arms races between hosts and parasites would not occur without genetic variation for traits involved in the outcome of parasitism. Genetic variations in resistance and virulence have only rarely been described in pairwise host-parasitoid interactions and have never been analysed in multi-species interactions, in contrast to well-characterized plant-pathogen interactions. This paper reports genetic variation in resistance of Drosophila yakuba to the parasitoid wasp Leptopilina boulardi. The genetic basis and geographic distribution of resistance is analysed. On the basis of these and previous findings, we demonstrate that there are
\end{abstract}

different resistance patterns to the parasitoid species $L$. boulardi in $D$. melanogaster and $D$. yakuba, as well as different specificity levels in the parasitoid species, suggesting complex ecological interactions in the field. This first description of resistance-virulence genetic interactions between a parasitoid and its two host species provides empirical data showing that multi-species interactions may greatly influence coevolutionary processes.

Heredity (2007) 98, 21-27. doi:10.1038/sj.hdy.6800893; published online 11 October 2006

Keywords: Drosophila; parasitoid wasp; resistance; geographical variation; genetic pattern of interaction; coevolution

\section{Introduction}

In host-parasite or host-pathogen interactions, antagonistic selective pressures can lead to a coevolutionary arms race based on adaptations and counter-adaptations in each partner. However, this process requires occurrence of genetic variation for traits involved in the final outcome of the interaction (Thompson, 1994; Sorci et al., 1997). Such variation has often been described in plantpathogen interactions (Frank, 1994), but little is known regarding interactions between insect parasitoids and their hosts. In this kind of interaction, where larval stages of the parasitoid are dependent on their arthropod host (Godfray, 1994), reciprocal selective pressures are particularly strong as only one partner can survive infestation.

To defend themselves against parasitoid attacks, host species have set up diverse behavioural and/or immune defences (Gross, 1993; Strand and Pech, 1995). In insects, the most well-known immune defence against parasitoids is the formation of a multi-cellular, melanized capsule around the parasitoid egg. This encapsulation reaction, when successful, leads to parasitoid death and host survival (Carton and Nappi, 1997). To counteract host immune defences, parasitoids have developed various strategies, based mainly on the use of virulence factors (Strand and Pech, 1995; Pennacchio and Strand,

Correspondence: $\operatorname{Dr}$ A Dubuffet, Faculté des Sciences et Techniques, Institut de Recherche sur la Biologie de l'Insecte, UMR CNRS 6035, Université François-Rabelais, Parc Grandmont, 37200 Tours, France.

E-mail:dubuffet@univ-tours.fr

Received 4 November 2005; revised 29 June 2006; accepted 12 July 2006; published online 11 October 2006
2006). Surprisingly, despite their relevance for the study of coevolution, little data are currently available on hostparasitoid interactions with regard to the genetic bases of resistance and virulence traits (Kraaijeveld et al., 1998; Dupas et al., 2002).

Variability in both resistance (ability to neutralize the parasitoid egg using immune defences) and virulence (ability to overcome the host immune defences) has been documented in only three host-parasitoid interactions, two of them involving the host species Drosophila melanogaster (Kraaijeveld and van Alphen, 1994; Dupas et al., 2002) and the other, the pea aphid Acyrthosiphon pisum (Henter, 1995; Henter and Via, 1995). In these models, genetic variation for both resistance and virulence has been observed and resistance and virulence components are thus predicted to coevolve under antagonistic selective pressures.

This interpretation is, however, unlikely to explain all resistance and virulence patterns in natural populations (Dupas et al., 2002). Indeed, as stressed by Thompson (1999), selective pressures exerted by other parasitoid and host species will also influence the geographic patterns of resistance-virulence in a given pairwise interaction. Unfortunately, data on resistance-virulence traits in complex interactions involving at least one parasitoid and different host species or one host species and its parasitoids are lacking. To estimate potential trade-offs or genetic constraints between resistance and virulence traits, it is now necessary to analyse several pairwise systems involving different interacting hostparasitoid species.

Drosophila species and their parasitoids represent one of the best models to analyse multiple species hostparasitoid interactions and to characterize resistance and 
virulence patterns in the field. Variability in resistance to two different parasitoids, Asobara tabida (Kraaijeveld and van Alphen, 1995) and Leptopilina boulardi (Dupas et al., 2002), has been observed in D. melanogaster, and variability in virulence has been reported in the parasitoid species L. boulardi against two Drosophila host species (Dupas and Boscaro, 1999). The genetic determinism of these traits has, however, only been studied in the D. melanogaster/L. boulardi pairwise interaction, using crosses between laboratory isofemale lines. In this system, simple genetic determinism was found, with one diallelic locus explaining resistance and one diallelic locus being responsible for virulence (Carton et al., 1992; Dupas et al., 1998). Interestingly, in tropical Africa, L. boulardi can infest several other species of the melanogaster subgroup of Drosophilidae, including $D$. yakuba (Carton and Nappi, 1991). This species is widespread on the African mainland (Lachaise et al., 1988) and was also described recently on some islands (Lachaise et al., 2000). Variability in virulence against D. yakuba has been observed in natural populations of L. boulardi and is also determined by a single diallelic locus (Dupas and Boscaro, 1999; Dupas and Carton, 1999).

The aim of this work was to quantify genetic variation for resistance in D. yakuba in natural populations and to analyse its genetic basis. We compared the genetic systems for resistance to $L$. boulardi in D. melanogaster and D. yakuba. Besides providing genetic information on a second pairwise host-parasitoid interaction, these data enable us to compare for the first time a parasitoid interaction with two different host species, thereby providing a complete picture of virulence-resistance traits in a multi-species host-parasite system.

Interestingly, combined data show that resistance patterns differ in closely related Drosophila host species and that different specificity levels can be observed in a single parasitoid species. Altogether, the L. boulardi/D. melanogaster interaction can be well explained by a 'genefor-gene' ('incompatibility') pattern, whereas interaction of $L$. boulardi with D. yakuba would be better described by a 'compatibility' pattern. Occurrence of genetically based variations of virulence and resistance in $L$. boulardi interactions with two different Drosophila host species makes this model unique for understanding the evolution of complex host-parasitoid relationships and addressing the question of the specificity of resistance and virulence traits.

\section{Materials and methods}

\section{Insects}

Reference lines of L. boulardi: $\quad$ L. boulardi isofemale lines IS $_{\mathrm{y}}$ and $\mathrm{IS}_{\mathrm{m}}$ have been described by Dupas et al. (1998). IS $_{\mathrm{m}}$ (Gif stock, no. 431) derives from a single female collected in the Nasrallah oasis (Tunisia). IS (Gif stock, no. 486) derives from a single female originating from a mass culture of a Brazzaville (Congo) population. $\mathrm{IS}_{\mathrm{m}}$ parasitoids are highly virulent against $D$. melanogaster but are always encapsulated in D. yakuba. On the contrary, parasitism success of IS $_{y}$ parasitoids in $D$. melanogaster depends on the resistance/susceptibility genotype of the flies. The $\mathrm{IS}_{\mathrm{y}}$ line was used by Carton et al. (1992) to study the genetics of resistance to $L$. boulardi in D. melanogaster.

Both $\mathrm{IS}_{\mathrm{m}}$ and $\mathrm{IS}_{\mathrm{y}}$ lines were reared on a susceptible D. melanogaster strain (Gif stock, no. 1333 ), at $25^{\circ} \mathrm{C}$. After emergence, adults of both lines were kept at $18^{\circ} \mathrm{C}$ on agar medium with honey.

Reference lines of $D$. yakuba: The isofemale lines 1880$\mathrm{D}\left(\mathrm{R}_{1}\right.$ line) and $1907\left(\mathrm{R}_{2}\right.$ line) were selected from two populations in Tanzania and further used to analyse the genetic basis of resistance. $R_{1}$ and $R_{2}$ show opposite immune capacities against the IS $_{\mathrm{y}}$ line of $L$. boulardi (see Table 1). These isofemale lines were obtained from a single inseminated founder female collected in the field (David et al., 2005) that was used to initiate a full sib line. The sib lines had been maintained in the laboratory for 8 years at the time of experiments, and were thus probably homozygous for all the loci potentially involved in resistance to the parasitoid wasp. The $R_{1}$ line was used by Dupas et al. (1998) to study the genetics of virulence of L. boulardi against D. yakuba.

Natural populations of D. yakuba: Seven D. yakuba populations were collected throughout the species distribution area, in the Afrotropical region, where $D$. melanogaster is also present (Lachaise et al., 1988). Several isofemale lines or multi-female strains were obtained from these populations. Occurrence of geographic variation in resistance to $L$. boulardi was tested using either multi-female strains or pools of 6-24 isofemale lines (Table 2). This method was previously shown to preserve the genetic variability of a population (David et al., 2005). All D. yakuba strains were raised at $25^{\circ} \mathrm{C}$ using a standard Drosophila cornmeal-yeast-agar medium.

\section{Bioassay procedure}

For bioassays, batches of 30 second instar D. yakuba larvae ( $48 \mathrm{~h}$ old) were submitted to parasitism by three $L$. boulardi females for $4 \mathrm{~h}$. All the bioassays were performed at $25^{\circ} \mathrm{C}$. Encapsulation ability was estimated $48 \mathrm{~h}$ later by dissecting late third instar larvae. At this time, a melanized capsule is found in resistant larvae, but not in susceptible ones. The encapsulation rate was calculated as the ratio of encapsulated parasitoid eggs to the number of parasitized hosts, using data from only monoparasitized larvae.

Table 1 Collection sites of the D. yakuba reference lines used for genetic analyses and percentage of encapsulation of $L$. boulardi eggs from the $\mathrm{IS}_{\mathrm{y}}$ and $\mathrm{IS}_{\mathrm{m}}$ lines

\begin{tabular}{|c|c|c|c|c|c|}
\hline $\begin{array}{l}\text { Strain number } \\
\text { (Gif stock) }\end{array}$ & Collection site & $\begin{array}{l}\text { Collection } \\
\text { year }\end{array}$ & Status & $\begin{array}{l}\text { Encapsulation rate of } I S_{y} \text { eggs } \\
\text { (number of larvae tested) }\end{array}$ & $\begin{array}{c}\text { Encapsulation rate of } I S_{m} \text { eggs } \\
\text { (number of larvae tested) }\end{array}$ \\
\hline $1880 \mathrm{D}\left(\mathrm{R}_{1}\right)$ & Tanzania, Udzungwa (south) & 1995 & Isofemale line & $18.8 \%(101)$ & $100 \%(89)$ \\
\hline $1907\left(\mathrm{R}_{2}\right)$ & Tanzania, Meru (north) & 1995 & Isofemale line & $92.9 \%(113)$ & $99.4 \%(157)$ \\
\hline
\end{tabular}


Table 2 D. yakuba strains collection sites, collection dates, status and percentage of encapsulation of L. boulardi eggs from the IS $\mathrm{y}_{\mathrm{y}}$ and $\mathrm{IS}_{\mathrm{m}}$ lines

\begin{tabular}{|c|c|c|c|c|c|}
\hline $\begin{array}{l}\text { Strain number } \\
\text { (Gif stock) }\end{array}$ & Collection site & Date & Status & $\begin{array}{l}\text { Encapsulation rate of } I S_{y} \text { eggs } \\
\text { (number of larvae tested) }\end{array}$ & $\begin{array}{c}\text { Encapsulation rate of } I S_{m} \text { eggs } \\
\text { (number of larvae tested) }\end{array}$ \\
\hline 1915 & Ivory Coast, Taï & 1983 & Multi-female strain & $77.6 \%(60)$ & $100 \%(57)$ \\
\hline 1919 & São Tome isle (hybrid zone) & 2001 & 7 isofemale lines & $7.5 \%(53)$ & $100 \%(77)$ \\
\hline 1917 & São Tome isle (south) & 2000 & 24 isofemale lines & $6.0 \%(84)$ & $100 \%(74)$ \\
\hline 1921 & Principe Isle & 2001 & 6 isofemale lines & $88.9 \%(54)$ & $100 \%(46)$ \\
\hline 1916 & Gabon, Lope & 1998 & 14 isofemale lines & $92.0 \%(50)$ & $100 \%(31)$ \\
\hline 1920 & Kenya, Mt Elgon & 1984 & Multi-female strain & $85.5 \%(55)$ & $100 \%(29)$ \\
\hline 1918 & Kenya, Mt Kenya & 1984 & Multi-female strain & $97.9 \%(47)$ & $100 \%(32)$ \\
\hline
\end{tabular}

Table 3 Percentage of encapsulation of L. boulardi $\mathrm{IS}_{\mathrm{y}}$ eggs by D. yakuba larvae originating from different crosses involving the $\mathrm{R}_{1}$ and $\mathrm{R}_{2}$ lines

\begin{tabular}{|c|c|c|c|c|}
\hline Crosses & Mother $\times$ father & Number of replicates & Number of larvae tested & Encapsulation rate (mean \pm s.e.) (\%) \\
\hline \multicolumn{5}{|c|}{ Parental lines } \\
\hline 1 & $\mathrm{R}_{1} \times \mathrm{R}_{1}$ & 13 & 240 & $12.5 \pm 2.1$ \\
\hline 2 & $\mathrm{R}_{2} \times \mathrm{R}_{2}$ & 14 & 299 & $86.0 \pm 2.0$ \\
\hline \multicolumn{5}{|c|}{ Reciprocal $F_{1}$ hybrids } \\
\hline 3 & $\mathrm{R}_{1} \times \mathrm{R}_{2}$ & 13 & 383 & $83.6 \pm 1.9$ \\
\hline 4 & $\mathrm{R}_{2} \times \mathrm{R}_{1}$ & 13 & 457 & $86.4 \pm 1.6$ \\
\hline \multicolumn{5}{|c|}{ Reciprocal $F_{2}$ hybrids } \\
\hline 5 & $\left(\mathrm{R}_{1} \times \mathrm{R}_{2}\right) \times\left(\mathrm{R}_{1} \times \mathrm{R}_{2}\right)$ & 11 & 419 & $62.5 \pm 2.4$ \\
\hline 6 & $\left(\mathrm{R}_{2} \times \mathrm{R}_{1}\right) \times\left(\mathrm{R}_{2} \times \mathrm{R}_{1}\right)$ & 12 & 370 & $60.0 \pm 2.6$ \\
\hline
\end{tabular}

\section{Genetic analysis}

Crossing procedure: Two generations of reciprocal crosses (Table 3 ) between the $R_{1}$ and $R_{2}$ lines, respectively susceptible and resistant to the parasitoid $I_{\mathrm{y}}$ line, were performed. The two parental lines, the two $\mathrm{F}_{1}$ hybrids and the two $\mathrm{F}_{2}$ hybrids were tested and compared for their encapsulation ability.

Statistical analysis: The mode of inheritance of $D$. yakuba resistance to the L. boulardi IS $_{\mathrm{y}}$ line was assessed using analyses of variance (ANOVA) with the generalized linear model procedure, assuming binomial error term distribution. Analyses were performed according to the method described by De Belle and Sokolowski (1987), which was previously used to analyse the genetic determinism of resistance of $D$. melanogaster to the parasitoid species $L$. boulardi and A. tabida (Carton et al., 1992; Benassi et al., 1998). The following comparisons were made (cross numbers refer to those described in Table 3): difference between parental strains ( 1 vs 2$)$, dominance or additive effects $(1+2$ vs $3+4)$, deviation from an autosomal mode of inheritance (3 vs 4).

The single-gene model with complete dominance of the resistance allele was tested using Mendelian analysis with the ratio of susceptible to resistant larvae, as described by Carton et al. (1992). The observed and expected ratios of susceptible to resistant were analysed using $\chi^{2}$ analysis. All statistical analyses were performed using Systat 10 software (SPSS Inc.).

\section{Results}

\section{Occurrence of variability in resistance of $D$. yakuba to}

\section{L. boulardi}

Two $D$. yakuba isofemale lines, $R_{1}$ and $R_{2}$, originating from Tanzanian populations, were selected from a range of candidate lines for their high $(92.9 \pm 2.4 \%)$ and low $(18.8 \pm 3.9 \%)$ encapsulation levels of eggs from the $L$. boulardi wasp IS $\mathrm{y}_{\mathrm{y}}$ line, respectively. Both lines encapsulated almost $100 \%$ of the eggs of the reference $\mathrm{IS}_{\mathrm{m}}$ line (99.4 and 100\%, respectively) (Table 1). Based on these results, $R_{1}$ and $R_{2}$ were considered as susceptible and resistant, respectively, to the $\mathrm{IS}_{\mathrm{y}}$ line of L. boulardi.

\section{Genetic determinism of resistance}

Encapsulation rates of IS $\mathrm{y}_{\mathrm{y}}$ parasitoid eggs by D. yakuba larvae originating from different crosses performed with the $R_{1}$ and $R_{2}$ isofemale lines, and results of the contrast analysis of variance are presented in Tables 3 and 4, respectively. As expected, the $R_{1}$ and $R_{2}$ lines differed significantly in their resistance to infestation by $\mathrm{IS}_{\mathrm{y}}$ parasitoids $(F=436.79, P<0.001) . \mathrm{F}_{1}$ hybrids exhibited significantly higher resistance than the mean parents, showing that resistance was dominant $(F=252.74$, $P<0.001)$. Reciprocal $F_{1}$ hybrids were not significantly different from each other, excluding the involvement of non-autosomal inheritance $(F=1.05, P=0.305)$. Larvae from the different crosses were classified into two classes (resistant and susceptible) according to their ability to encapsulate IS $\mathrm{y}_{\mathrm{y}}$ eggs. The single gene model hypothesis was tested by comparing susceptible vs resistant proportions (S:R) with expected Mendelian proportions, using $\chi^{2}$ analysis. The results (Table 5) were consistent with the hypothesis of a single major segregating locus with two alleles, the resistant one being completely dominant. The locus responsible for resistance of $D$. yakuba to $\mathrm{IS}_{\mathrm{y}}$ parasitoids was named $R \mathrm{lb}_{I S y}$ (for resistance to L. boulardi of the IS $\mathrm{S}_{\mathrm{y}}$ type) and the resistant and susceptible alleles, $R l b_{I S y}^{+}$and $R l b_{I S y}$, respectively.

\section{Genetic variability in natural populations}

Encapsulation rates of the two reference $L$. boulardi lines, $\mathrm{IS}_{\mathrm{y}}$ and $\mathrm{IS}_{\mathrm{m}}$, were recorded in seven $D$. yakuba 
Table 4 Contrast ANOVA for encapsulation of L. boulardi $\mathrm{IS}_{\mathrm{y}}$ eggs by D. yakuba larvae originating from crosses involving the $\mathrm{R}_{1}$ and $\mathrm{R}_{2}$ lines (generalized linear model with binomial error term)

\begin{tabular}{lrrr}
\hline Source & d.f. & MS & F \\
\hline Model (between crosses) & 5 & 22.38 & 136.10 \\
Contrasts & & & $<0.001$ \\
1. R R $_{2}$ parental lines (1 vs 2) & 1 & 71.83 & 436.79 \\
2. Dominance (1+2 vs 3+4) & 1 & 41.56 & $<0.001$ \\
3. Deviation from an autosomal mode of inheritance (3 vs 4) & 1 & 0.17 & 252.74 \\
Error (within crosses) & 2162 & 0.164 & 1.05 \\
\hline
\end{tabular}

Table 5 Deviation of the proportions of (susceptible vs resistant) (S:R) larvae in the progeny of different crosses from the proportions expected under a one-locus model with dominance of the resistance allele

\begin{tabular}{|c|c|c|c|c|c|c|}
\hline & $\begin{array}{c}\text { Expected S:R proportion } \\
\text { under the model }\end{array}$ & $\mathrm{N}$ & $\begin{array}{l}\text { Expected number of } S: R \\
\text { larvae under the model }\end{array}$ & Observed number of S:R larvae & $\chi^{2}$ & $\mathrm{P}$ \\
\hline \multicolumn{7}{|c|}{ Parental lines } \\
\hline $\mathrm{R}_{1} \times \mathrm{R}_{1}$ & 1:0 & 240 & $240: 0$ & 210:30 & & \\
\hline \multirow[t]{2}{*}{$\mathrm{R}_{2} \times \mathrm{R}_{2}$} & $0: 1$ & 299 & $0: 299$ & $42: 257$ & & \\
\hline & & & & $\begin{array}{l}\text { Allows calculating the } \\
\text { misclassification level }\end{array}$ & & \\
\hline \multicolumn{7}{|c|}{ Reciprocal crosses } \\
\hline \multicolumn{7}{|c|}{$\mathrm{F}_{1}$} \\
\hline $\mathrm{R}_{1} \times \mathrm{R}_{2}$ & $0: 1$ & 383 & $\begin{array}{c}0: 383 \\
\text { Corrected: } 51: 332\end{array}$ & $63: 320$ & 3.26 & $0.07 \mathrm{NS}$ \\
\hline $\mathrm{R}_{2} \times \mathrm{R}_{1}$ & $0: 1$ & 457 & $\begin{array}{c}\text { 0:457 } \\
\text { Corrected: } 61: 396\end{array}$ & $62: 395$ & 0.02 & $0.89 \mathrm{NS}$ \\
\hline \multicolumn{7}{|l|}{$\mathrm{F}_{2}$} \\
\hline $\mathrm{F}_{1} \times \mathrm{F}_{1}$ & $1: 3$ & 789 & $\begin{array}{c}\text { 197:592 } \\
\text { Corrected: } 302: 487\end{array}$ & $305: 484$ & 0.05 & $0.83 \mathrm{NS}$ \\
\hline
\end{tabular}

About 12.5\% (30 out of 240) of larvae of the 'susceptible' $R_{1}$ parental line encapsulate L. boulardi eggs, whereas 14\% (42 out of 299) of larvae of the 'resistant' $\mathrm{R}_{2}$ parental line fail to encapsulate these eggs. This constant observed deviation from the expected 0 and $100 \%$ encapsulation rates is mainly due to environmental effects. This corresponds to a mean misclassification rate of 13.25\%. The expected number of S:R larvae in the progeny of the crosses was thus corrected to include this misclassification factor, according to the method of Carton et al. (1992) and Benassi et al. (1998). Data were then compared using a $\chi^{2}$ goodness of fit test. NS: nonsignificant.

populations (Table 2). Figure 1 presents the geographic pattern of resistance to IS parasitoids.

A high level of variation in resistance to the $\mathrm{IS}_{\mathrm{y}}$ line was observed in the field, which confirmed the results obtained with the Tanzanian $R_{1}$ and $R_{2}$ isofemale lines: resistance to IS y $_{\mathrm{y}}$ in . yakuba shows significant variability. Encapsulation rates varied from 6 to $98 \%$ but most populations encapsulated IS $\mathrm{y}_{\mathrm{y}}$ eggs at a high frequency (from 77.6 to $97.9 \%$ ). Only populations from the São Tome island were susceptible to IS $_{\mathrm{y}}$ infestation (6.5 and $7.0 \%$ of IS $_{y}$ eggs encapsulated). Resistance was thus found either at a very high or a very low frequency in D. yakuba Afrotropical populations. By contrast, there was no variability between $D$. yakuba strains for resistance to the $\mathrm{IS}_{\mathrm{m}}$ parasitoid line, with $100 \%$ of eggs encapsulated in each strain (Table 2). Despite intense investigations, no $D$. yakuba population susceptible to the $\mathrm{IS}_{\mathrm{m}}$ line of $L$. boulardi has ever been found.

\section{Discussion}

\section{Genetic determinism of parasitoid resistance in Drosophila}

The present study reports occurrence of variation in the ability of $D$. yakuba to encapsulate eggs of the IS $_{\mathrm{y}}$ line of $L$. boulardi. Using crosses between lines having

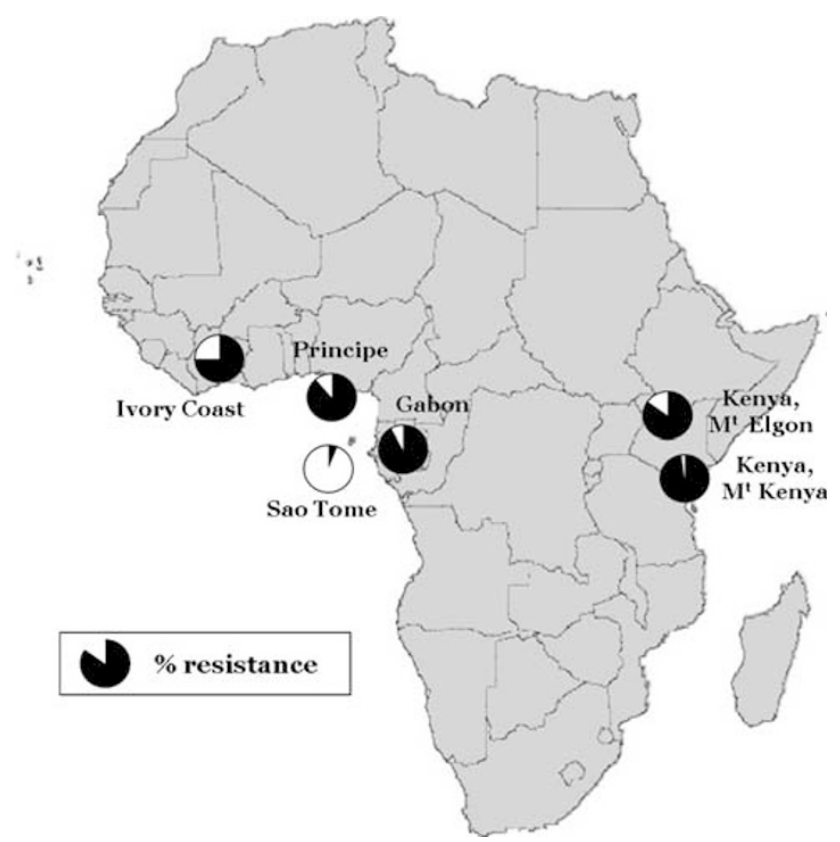

Figure 1 Geographical distribution of $D$. yakuba resistance to IS $_{\mathrm{y}}$ parasitoids in tropical Africa. The resistance level (in black) was estimated from the percentage of IS $_{\mathrm{y}}$ eggs encapsulated by $D$. yakuba larvae. 
contrasted resistance levels, we demonstrate that resistance is determined by a single major locus, which we termed $R l b_{I S y}$.

Considering the high number of genes involved in insect immune pathways (Irving et al., 2001; Zettervall et al., 2004), variation in resistance to parasitoid wasps was expected to be determined by multigenic systems (Sorci et al., 1997). However, in all cases in which genetic determinism of resistance to parasitoids has been studied - D. melanogaster/L. boulardi, D. melanogaster/A. tabida and now $D$. yakuba/L. boulardi - differences between lines resistant and susceptible to a given parasitoid have always been explained by a single diallelic locus. In $D$. melanogaster, two loci, named Rat and Rlb (labelled as $R s t(2) L b$ in Flybase; ID number: FBgn0016729), localized 35 centimorgans apart, are responsible for resistance to the parasitoids $A$. tabida and $L$. boulardi, respectively (Carton et al., 1992; Benassi et al., 1998; Poirié et al., 2000). The use of isofemale lines, which are not representative of the extent of genetic variation in the field, might favour the recovery of simple genetic systems (Kraaijeveld et al., 1998). However, studies by Orr and Irving (1997), dealing with genetic variation of resistance to $A$. tabida in $D$. melanogaster populations from different parts of Europe, also concluded on a simple genetic basis for resistance. The advantage of performing analyses with well-characterized strains is that it allows the precise localization and identification of resistance loci (Hita et al., 1999; M Poirié, unpublished data). These loci are expected to contain genes involved in the response to parasitoid attacks, showing enough polymorphism to respond to selection pressures and thus potentially evolving under coevolutionary processes.
The genetic bases of interactions between two different lines of $L$. boulardi and the two host species D. melanogaster and D. yakuba are summarized in Figure 2. It is the first complete report of genetic interactions between a parasitoid and two different host species.

In the L. boulardi-D. melanogaster system, the success of parasitism is the rule; failure only occurs when the parasitoid has no virulence alleles $\left(I S_{m}^{-} / I S_{m}^{-}, I_{\mathrm{y}}\right.$ line) and when the host is resistant (at least one $R l b^{+}$allele, resistant strain) (Dupas et al., 2002). This system resembles the 'gene-for-gene' (or 'incompatibility') model of plant-pathogen interactions (Briggs and Johal, 1994). Here one parasitoid genotype ( $\mathrm{IS}_{\mathrm{m}}$ line) has a 'universal virulence' (Frank, 1994), which means that it can infest all D. melanogaster flies, whatever their genotypes.

By contrast, the general outcome in the L. boulardi$D$. yakuba interaction is the encapsulation of the parasite egg; parasitism is successful only if the parasitoid is homozygous for virulence alleles $\left(I S_{y}^{+} / I S_{y}^{+}, I S_{y}\right.$ line) (Dupas et al., 1998) and if the host is homozygous for susceptible alleles $\left(R l b_{\overline{I s y}} / R l b_{\overline{I s} \text {, }}, \mathrm{R}_{1}\right.$ line). This genetic pattern of interactions, different from the one described above, resembles the 'compatibility model' of Briggs and Johal (1994). In this model, parasitoid success would require a specific match between host and parasite 'compatible factors' and each modification of the host target would prevent development of the parasite.

Genetic variations in resistance and virulence have rarely been described in pairwise host-parasitoid interactions and had never been analysed before in multispecies interactions. Our data show that there are different resistance patterns to the parasitoid species

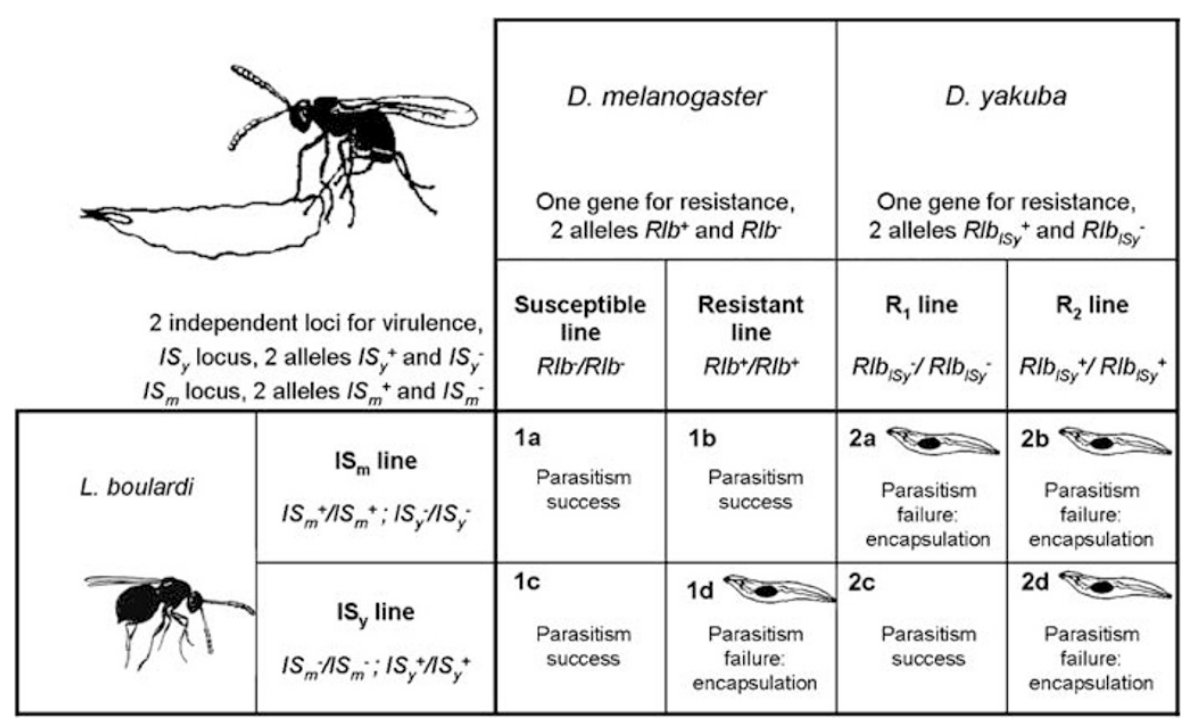

Figure 2 Genetic interactions matrix in the Drosophila-L. boulardi reference system. The interactions between the reference lines IS $\mathrm{y}_{\mathrm{y}}$ and $\mathrm{IS}_{\mathrm{m}}$ of the parasitoid L. boulardi and the reference lines of the host species D. melanogaster and D. yakuba are illustrated. Resistance in each host species is conferred by one major locus, and two independent loci are responsible for virulence of the parasitoid against the two host species. In the L. boulardi-D. melanogaster system (cells 1a to $1 \mathrm{~d}$ ), the host alleles $\mathrm{Rlb}^{+}$and $\mathrm{Rlb}^{-}$are responsible for resistance and susceptibility to IS infection, and the parasitoid alleles $I S_{m}^{+}$and $I S_{m}^{-}$are responsible for virulence and avirulence of the parasitoid against the host. Parasitism success is the rule in D. melanogaster (cells $1 \mathrm{a}, 1 \mathrm{~b}$ and $1 \mathrm{c}$ ), except when a resistance allele $R l b^{+}$is present without any virulence allele $I S_{m}^{+}$(cell 1d). In the $L$. boulardi-D. yakuba system (cells $2 \mathrm{a}$ to $2 \mathrm{~d}$ ), the host alleles $R l b_{I S y}$ and $R l b_{\overline{I S}}$ are responsible for resistance and susceptibility to the $\mathrm{IS}_{\mathrm{y}}$ infection, and the parasitoid alleles $I S_{y}^{+}$and $I S_{y}^{-}$are responsible for virulence and avirulence of the parasitoid against the host $\mathrm{R}_{1}$ line. In this system, parasitism failure is the rule (cells $2 \mathrm{a}, 2 \mathrm{~b}$ and $2 \mathrm{~d}$ ), except when two virulence alleles $I S_{y}^{+}$are present with two susceptibility alleles $R l b_{\overline{I S} y}$ (cell 2c). 
L. boulardi in D. melanogaster and D. yakuba, suggesting complex ecological interactions in the field.

$\mathrm{IS}_{\mathrm{m}}$ eggs are rarely encapsulated in $D$. melanogaster and always encapsulated in D. yakuba lines. Despite intense investigations, we have not been able to recover $D$. melanogaster flies that are resistant or D. yakuba flies that are susceptible to this parasitoid line. By contrast, encapsulation of $\mathrm{IS}_{\mathrm{y}}$ eggs can take place in both host species depending on the genotype of the fly (ie the resistance status of the host strain). Hence, the success of the $I S_{m}$ line is 'species-dependent', whereas the success of the $I_{\mathrm{y}}$ line is 'host-genotype-dependent'. We thus report the occurrence of two different levels of host specificity in a single parasitoid species. As suggested in other host-parasite systems, the success or failure of a parasitoid seems to be due to neither parasitoid virulence alone nor host resistance ability alone, but rather determined by complex interactions between host and parasitoid species (Kraaijeveld and Godfray, 2001; Dupas et al., 2002; Little et al., 2005).

\section{Geographic patterns of resistance of $D$. yakuba to}

\section{L. boulardi}

L. boulardi eggs of the $\mathrm{IS}_{\mathrm{m}}$ line were encapsulated in all D. yakuba strains tested, whatever their geographic origin, thus confirming the absence of variation in resistance to this parasitoid line. Occurrence of variability in resistance to IS $_{\mathrm{y}}$ females, discovered in Tanzanian populations (origin of the $R_{1}$ and $R_{2}$ lines), was further confirmed using other natural populations. The question remains whether this variability in resistance can also be attributed to the $R l b_{I S y}$ locus.

A high resistance level was found in Kenya, Gabon, Ivory Coast and in the Principe Island, whereas populations from São Tome were susceptible. Interestingly, resistance to IS $_{\mathrm{y}}$ parasitoids is also high in D. melanogaster populations, even in areas where this type of parasitoid is absent (Dupas et al., 2002). It is generally postulated that resistance to a pathogen or a parasite is a life-history trait whose polymorphism is maintained by a balance between positive selection forces (the presence of the pathogen) and counter-selecting forces (the cost of maintaining resistance in the absence of the pathogen) (Kurtz et al., 2002; Rolff and Siva-Jothy, 2003). In this model, positive selective forces should have acted in every locality where resistance to IS $_{\mathrm{y}}$ parasitoids is observed at high frequencies. So far, L. boulardi popula-

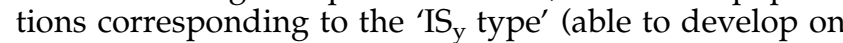
D. yakuba) have only been found in Congo and, to a lesser extent, in Ivory Coast (Dupas and Boscaro, 1999). If the

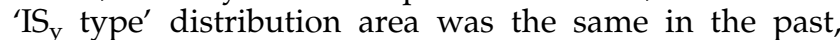
other biotic or abiotic pressures might have selected resistance in the majority of $D$. yakuba populations. Whether individuals resistant to 'IS $\mathrm{S}_{\mathrm{y}}$ parasites' can also resist other parasitoids/pathogens remains to be determined, but preliminary results suggest that they are at least able to resist another parasitoid species found in these localities, Leptopilina freyae (Allemand et al., 2002; Y Carton, unpublished data). Resistance in D. yakuba populations could be considered in this way to be a 'generalist resistance' rather than a 'specific resistance'.

According to the same hypothesis, the absence of resistance in São Tome island could result from the absence of agents selecting resistance in mainland populations, combined with counter-selection of resistance alleles, leading to the loss of resistance. Unfortunately, the questions whether L. boulardi parasitoids can be found in São Tome and whether D. yakuba resistance is costly remain to be answered. A second hypothesis could be that under a high diversity of attack or a high prevalence of parasites, tolerance or non-investment in immune defence mechanisms is an optimal strategy (Sasaki and Godfray, 1999; Jokela et al., 2000). We lack information on the abundance of $D$. yakuba pathogens or parasitoids on this island but considering classical theories in biogeography, this hypothesis seems rather unlikely (MacArthur and Wilson, 1967). Finally, the susceptibility of São Tome populations to L. boulardi might be explained by a founder effect having occurred during colonization of the island by a small number of flies (Cariou et al., 2001).

Observation of variability in resistance and virulence in host-parasitoid communities and determination of their genetic bases are important in understanding diffuse coevolutionary processes. This can be achieved by analysing and comparing pairwise interactions involving the same parasitoid and different host species or the same host and different parasitoid species. We draw here the first complete picture of resistance-virulence genetic interactions between a parasitoid and its two host species, and suggest that multi-species interactions may indeed greatly influence coevolutionary processes. Deciphering the physiological and molecular bases of these traits will finally help to address the question of specificity in host-parasitoid interactions and will provide precious information on the ongoing coevolutionary arms races.

\section{Acknowledgements}

This work was supported by the French GDR 2153. Aurore Dubuffet received financial support from the François Rabelais University. We are grateful to Daniel Lachaise for providing Drosophila lines, to Carole Labrousse for technical assistance and to Elisabeth Huguet and Georges Periquet for helpful comments on the manuscript.

\section{References}

Allemand R, Lemaître C, Frey F, Boulétreau M, Vavre F, Nordlander G et al. (2002). Phylogeny of six African Leptopilina species (Hymenoptera: Cynipoidea, Figitidae), parasitoids of Drosophila, with description of three new species. Ann Soc Entomol Fr 38: 319-332.

Benassi V, Frey F, Carton Y (1998). A new specific gene for wasp cellular immune resistance in Drosophila. Heredity $\mathbf{8 0}$ : 347-352.

Briggs SP, Johal GS (1994). Genetic patterns of plant-parasite interactions. Trends Genet 10: 12-16.

Cariou ML, Silvain JF, Daubin V, Da Lage JL, Lachaise D (2001). Divergence between Drosophila santomea and allopatric or sympatric populations of D. yakuba using paralogous amylase genes and migration scenarios along the Cameroon volcanic line. Mol Ecol 10: 649-660.

Carton Y, Frey F, Nappi AJ (1992). Genetic determinism of the cellular immune reaction in Drosophila melanogaster. Heredity 69: 393-399.

Carton Y, Nappi AJ (1991). The Drosophila immune reaction and the parasite capacity to evade it: genetic and coevolutionary aspects. Acta Oecol 12: 89-104. 
Carton Y, Nappi AJ (1997). Drosophila cellular immunity against parasitoids. Parasitol Today 13: 218-227.

David JR, Gibert P, Legout H, Petavy G, Capy P, Moreteau B (2005). Isofemale lines in Drosophila: an empirical approach to quantitative trait analysis in natural populations. Heredity 94: 3-12.

De Belle S, Sokolowski MB (1987). Heredity of rover/sitter: alternative foraging strategies of Drosophila melanogaster larvae. Heredity 59: 73-83.

Dupas S, Boscaro M (1999). Geographic variation and evolution of immune suppressive genes in a Drosophila parasitoid. Ecography 22: 284-291.

Dupas S, Carton Y (1999). Two non-linked genes for specific virulence of Leptopilina boulardi against Drosophila melanogaster and D. yakuba. Evol Ecol 13: 211-220.

Dupas S, Carton Y, Poirié M (2002). The genetic dimension of the coevolution of virulence resistance in Drosophila-parasitoid wasp relationships. Heredity 90: 84-89.

Dupas S, Frey F, Carton Y (1998). A single parasitoid segregating factor controls immune suppression in Drosophila. J Hered 89: 306-311.

Frank SA (1994). Recognition polymorphism in host-parasite genetics. Philos Trans R Soc London B 346: 283-293.

Godfray HCJ (1994). Parasitoids: Behavioral and Evolutionary Ecology. University Press: Princeton.

Gross P (1993). Insect behavioral and morphological defenses against parasitoids. Annu Rev Entomol 38: 251-273.

Henter HJ (1995). The potential for coevolution in a hostparasitoid system. II. Genetic variation within a population of wasps in the ability to parasitize an aphid population. Evolution 49: 439-445.

Henter HJ, Via S (1995). The potential for coevolution in a host-parasitoid system. I. Genetic variation within an aphid population in susceptibility to a parasitic wasp. Evolution 49: 427-438.

Hita MT, Poirié M, Leblanc N, Lemeunier F, Lutcher F, Frey F et al. (1999). Genetic localization of a Drosophila melanogaster resistance gene to a parasitoid wasp and physical mapping of the region. Genome Res 9: 471-481.

Irving $\mathrm{P}$, Troxler L, Heuer TS, Belvin M, Kopczynski C, Reichhart JM et al. (2001). A genome-wide analysis of immune responses in Drosophila. Proc Natl Acad Sci USA 98: 15119-15124.

Jokela J, Schmid-Hempel P, Rigby MC (2000). Dr Pangloss restrained by the Red Queen - steps towards an unified defence theory. Oikos 89: 267-274.

Kraaijeveld AR, Godfray HCJ (2001). Is there local adaptation in Drosophila-parasitoid interactions? Evol Ecol Res 3: 107-116.

Kraaijeveld AR, van Alphen JJ, Godfray HC (1998). The coevolution of host resistance and parasitoid virulence. Parasitology 116: S29-S45.
Kraaijeveld AR, van Alphen JJM (1994). Geographical variation in resistance of the parasitoid $A$. tabida against encapsulation by $D$. melanogaster larvae: the mechanism explored. Physiol Entomol 19: 9-14.

Kraaijeveld AR, van Alphen JM (1995). Geographical variation in encapsulation ability of Drosophila melanogaster larvae and evidence for parasitoid specific components. Evol Ecol 9: 10-17.

Kurtz J, van der Veen IT, Ryder JJ (2002). Ecological immunity of arthropods - a thread of Ariadne? Trends Ecol Evol 17: 204-205.

Lachaise D, Cariou M-L, David JR, Lemeunier F, Tsacas L, Ashburner M (1988). Historical biogeography of the Drosophila melanogaster species subgroup. In: Hecht M, Wallace B, Prance GT (eds). Evolutionary Biology, Vol 22. Plenum Publishing Corporation: New York, pp 159-225.

Lachaise D, Harry M, Solignac M, Lemeunier F, Bénassi V, Cariou M-L (2000). Evolutionary novelties in islands: Drosophila santomea, a new melanogaster sister species from São Tomé. Proc R Soc London B 267: 1487-1495.

Little TJ, Hultmark D, Read AF (2005). Invertebrate immunity and the limits of mechanistic immunology. Nat Immunol 6: 651-654.

MacArthur RH, Wilson EO (1967). The Theory of Island Biogeography. Princeton University Press: Princeton, NJ.

Orr HA, Irving S (1997). The genetics of adaptation - the genetic basis of resistance to wasp parasitism in Drosophila melanogaster. Evolution 51: 1877-1885.

Pennacchio F, Strand MR (2006). Evolution of developmental strategies in parasitic hymenoptera. Annu Rev Entomol 51: 233-258.

Poirié M, Frey F, Hita M, Huguet E, Lemeunier F, Periquet G et al. (2000). Drosophila resistance genes to parasitoids: chromosomal location and linkage analysis. Proc $R$ Soc London B 267: 1417-1421.

Rolff J, Siva-Jothy MT (2003). Invertebrate ecological immunology. Science 301: 472-475.

Sasaki A, Godfray HCJ (1999). A model for the coevolution of resistance and virulence in coupled host-parasitoid interactions. Proc $R$ Soc London B 266: 455-463.

Sorci G, Møller AP, Boulinier T (1997). Genetics of host-parasite interactions. Trends Ecol Evol 12: 196-200.

Strand MR, Pech LP (1995). Immunological basis for compatibility in parasitoid-host relationships. Annu Rev Entomol 40: 31-56.

Thompson JN (1994). The Coevolutionary Process. University of Chicago Press: Chicago.

Thompson JN (1999). Specific hypotheses on the geographic mosaic theory of coevolution. Am Nat 153: S1-S14.

Zettervall C-J, Anderl I, Williams MJ, Palmer R, Kurucz E, Andó I (2004). A directed screen for genes involved in Drosophila blood cell activation. Proc Natl Acad Sci USA 101: 14192-14197. 